\title{
Research on Multi-service Unified Bearing Electric Power Communication Access Network
}

\author{
Bao Feng ${ }^{1, a}$, Yang $\mathrm{Li}^{1}$, Yang $\mathrm{Hu}^{1}$, Yan Long ${ }^{2}$, Yongzhong $\mathrm{Xie}^{3}$ \\ ${ }^{1}$ Department of Information and Communication, State Grid Electric Power Research Institute, \\ Nanjing, 210003, China \\ ${ }^{2}$ Department of Binhai Power Supply Branch, State Grid Tianjin Electric Power Company, Tianjin, \\ 300450, China \\ ${ }^{3}$ Department of Xuzhou Power Supply Branch, State Grid Jiangsu Electric Power Company, \\ Xuzhou, 221000, China \\ aemail: fengbao@sgepri.sgcc.com.cn
}

Keywords: Smart Grid; Security Region; Multi-service Isolation; QoS; EPON

\begin{abstract}
With the development of smart grid, especially the energy Internet, the emphasis on electric power communication network construction is gradually transferring from backbone level to access level. As a direct user-oriented network, electric power communication access network need to have features of wide area coverage, reliable transport, convenient maintenance, reasonable cost and so on. However, the current constructed access network is independent from each other because of security and isolation requirements. This led to many problems, such as network structure is unreasonable, communication resources are scattered and difficulty to share, and redundant construction. In order to solve those problems, we proposed a novel multi-service unified bearing network scheme based on key technology such as multi-service isolation and QoS. And implementation scheme based on EPON is also put forward. Simulation provide the proposed scheme is effective. Compared with the current network, the proposed scheme can improve the performance of electric power communication access network from multiple dimensions, and it is a direction of the future.
\end{abstract}

\section{Introduction}

In the past few years, development process of smart grid is accelerating. As the basic supporting system for smart grid, electric power communication network also got rapid development. The communication network of state grid is composed of backbone network and access network [1]. In the backbone level, the architecture of OTN + PTN or OTN + SDH is mainly used, which can basically meet the needs of long-distance transmission of large granular service. In the access level, it is an EPON, industrial Ethernet, PLC and wireless technology hybrid communication network, which can meet needs of a variety of application scenarios.

As a special used network, electric power communication network is mainly used to carry power production \& scheduling service and management $\&$ information service that belongs to different security region [2]. According to power monitoring system security protection regulations from national development and reform commission, services from different security region must realize physical isolation, other must realize logical isolation. SDH technology can realize physical isolation with standalone time slot. It can realize all electric power communication services bearing in one physical network. But packet switching technology is mainly used in the access level, which can only achieve logic isolation. It cannot bear different security region's services in one network. In order to bear all services, we must construct two physical networks, even the geographical distribution is coincidence. This has led to a lot of redundant construction, which caused a huge waste of construction and OAM costs. In order to reduce network costs, a novel scheme of multi-service unified bearing network is proposed. 


\section{The Current Electric Power Communication Access Network}

According to different supporting objects, electric power communication access network consist of power distribution and electricity consumption communication network. The voltage level of power distribution communication network is $10 \mathrm{kV}$, which is mainly used for distribution automation service bearing, telecommunication channel of electricity consumption information collection, uplink channel of intelligent community, and so on. The voltage level of electricity consumption communication network is $0.4 \mathrm{kV}$, which is mainly used for local channel of electricity consumption information collection, intelligent community, and so on. In the past five years, communication coverage and optical cable length continues improve. And it has gradually formed a fiber optic based, wireless and PLC supplemented, kinds of technologies hybrid networking scheme.

Depending on the transmission medium, communication technology of electric power communication access network mainly include optical fiber, wireless and power line carrier. As a typical optical fiber communication technology, EPON has widely used for service which had higher requirements on real-time, reliability and security [3]. Industrial Ethernet mainly used for feeder automation service bearing, it is also an optical fiber communication technology. There are two kind wireless communication networks: private and public. At present, the private wireless network mainly used LTE technology in $230 \mathrm{MHz}$ frequency band for load control service, and in $1.8 \mathrm{GHz}$ frequency band for all services. WiMAX and McWiLL also have small scale application. GPRS and CDMA are regularly used in the public wireless network for electricity consumption information gathering system. PLC is mainly including MV-PLC (medium voltage PLC) for a supplement to power distribution network, and LV-PLC (low voltage PLC) for electricity consumption information gathering system.

According to the type of services bearing, electric power communication network can be dividing into two types: power distribution network and electricity consumption network [4]. Power distribution communication network is mainly bearing service of distribution automation system, power quality monitoring, distributed power access $(\geqslant 200 \mathrm{~kW})$ and video surveillance of power distribution. Electricity consumption communication network is mainly bearing service of electricity consumption information collection, electric vehicle charging stations, distributed power access $(<200 \mathrm{~kW})$ and power quality monitoring (low voltage side). Service of electric power communication access network can be dividing into two security region: production control (include safety zone I and II) and management information (include safety zone III and IV). To ensure security of network, service of the same security region need to implement logical isolation (e.g. safety zone I and II), and service of different security region need to implement physical isolation (e.g. safety zone I and III).

According to network construction status, communication technology status and service bearing status, we can find it has formed coexist status of multi-service, multi-network and multiple communication technology, which is shown in Fig.1. That will cause many problems, such as: seriously repeat construction leads to high cost, utilization of the network is low, reliability of the network is low, new services deployment is not flexible. 


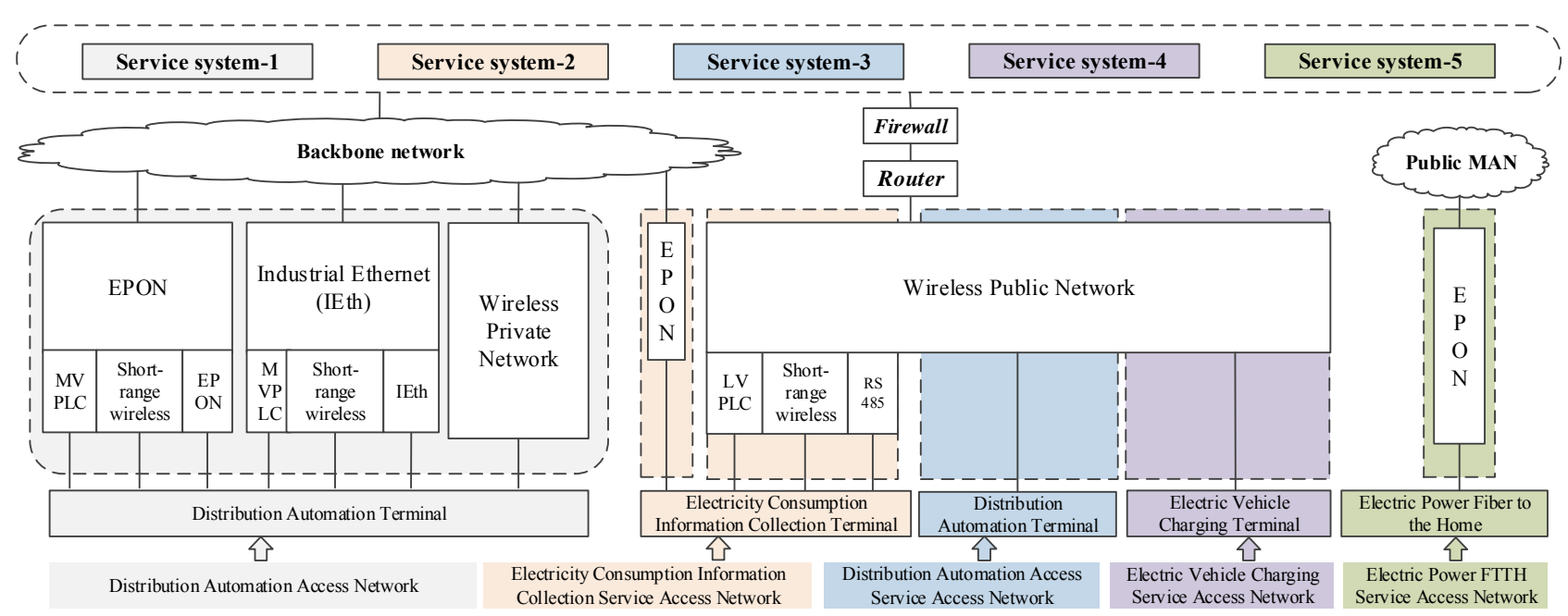

Fig.1. The architecture of current electric power communication access network

\section{Key Supporting Technology of Muti-service Bearing Network}

In order to support multi-service unified bearing, we must take into account problems of bearing service in different security region with one physical network. It mainly includes multi-service isolation and QoS supporting technology.

\section{Multi-service Isolation Technology}

There are two types of multi-service isolation technologies: logical isolation and physical isolation. Logical isolation is mainly for services from one security region, such as safety zone I and II, or safety zone III and IV. Physical isolation is mainly for services from different security region.

For logical isolation, the typical technology is VLAN [5]. Every VLAN is a broadcast domain. Due to in different broadcast domain, it cannot directly communicate in different VLANs. There are many methods for VLAN division, which is including port, MAC, protocol, subnet and so on. Service distinguish method of VLAN is only through add VLAN ID in the frame, that is to say, various services are still jointly sharing the communication resources at the same time. In the electric power communication access network, multi-service bearing can be realized by assigning all the same security region services in one VLAN.

For physical isolation, methods approved by state grid are including different fiber, different wavelength, and different time slot. Different fiber method. Two completely independent communication systems with different equipment and fiber are used to realize physical isolation of different security region. Therefore, it is complete physical isolation, but the construction cost is very high, which is almost the same as the current individual network. Different wavelength method. Two wavelengths that no interference between each other is used for bearing services from different security regions. It has higher cost compared with single wavelength communication system, but lower cost than different fiber method. Different time slot method. Different from the above two methods, time slot method adopts the same fiber and the same wavelength to bear different security region services, typical as $\mathrm{SDH}$.

\section{QoS Technology}

QoS refers to a network that can use a variety of basic technology to provide a better service for the communication network. And it can be used to solve the problem of delay, congestion and so on. In order to realize QoS mechanism, we should do flow classifying, policing and marking at the entry port, queuing and scheduling at the exit port. Line rating and traffic shaping are the key technologies to guarantee network traffic controllable and predictable. As an example, different stream flow control technologies are adopted in the uplink and downlink of EPON network for different QoS requirements. In the downlink, QoS mechanism of EPON is similar to Ethernet. In the uplink, the key QoS implementation technique of EPON is DBA (Dynamically Bandwidth Assignment) [6]. SBA (Static Bandwidth Allocation) is also used. 


\section{The Unified Multi-service Bearing Network Scheme Based on EPON}

\section{Unify Network Architecture}

The multi-service bearing electric power communication access network will adopt unify network architecture, which is shown in Fig.2.



Fig.2. Architecture of multi-service unified bearing network

As shown in Fig.2, the multi-service unified bearing electric power communication access network is mainly composed of terminal communication equipment with multi-service access function, core communication equipment with multi-uplink ports, and service terminal with service access. Both terminal and core communication equipment adopt multi-service isolation and communication performance safeguard technology, i.e. QoS and so on. Isolation almost physic is realized inside the equipment by certain means. Using the above technology and network architecture, we can construct one physical network that can carry all service in the access layer. The backbone network and service system will keep the original status. Due to the most commonly used communication technology of electric power communication access network is EPON, so we will mainly focus on EPON in the following sections.

\section{Multi-service Isolation Scheme of EPON}



Fig.3. Multi-service isolation scheme of EPON

As shown in Fig.3, several isolation technologies are used in the entire process of communication. Service terminal level. Different service terminals are connected to different communication port of ONU. ONU level. Firstly, service flows can be identified by ONU based on communication port. Security region that communication port belongs to can configure by NMS. Then, fixed size buffer resource is allocated for each security region using buffer cache resource partitioning technology. So services of different security regions cannot affect with each other. Fiber channel level. Different time slot or wavelength is used to realize channel isolation. Fixed and cyclically appeared time slot is allocated by using LLID for each ONU uplink channel. In downlink 
channel adopts time slot broadcast mode, fixed time slot only broadcast to corresponding service security region. OLT level. Firstly, similar to ONU, services identify and multi-buffer cache technologies are used for service aggregation and separation. Then, queue scheduling technology based on fixed time slot is used, within the specified time slot only exchange service of specified security region. In order to prevent MAC address attack between different security regions, multiple MAC table is used. At last, the already exchanged services of different security region enter into the corresponding security region buffer cache with an individual uplink port connected to backbone network. Backbone network level. Different service flows are transported to master station through the different physical network. From the above scheme, we can implement almost physical isolation of services from different security region.

\section{QoS Scheme of EPON}

As shown in Fig.4, QoS scheme of multi-service unified bearing electric power communication access network with EPON is mainly same as the current single service one, except fixed resources allocation for control service flow. The control service flow enjoys the highest priority. The other services will share resources according to importance degree. For the services of production control security region, it will obtain fixed time slot which is similar to SDH. For the service of management information security region, it will use DBA for bandwidth allocation.

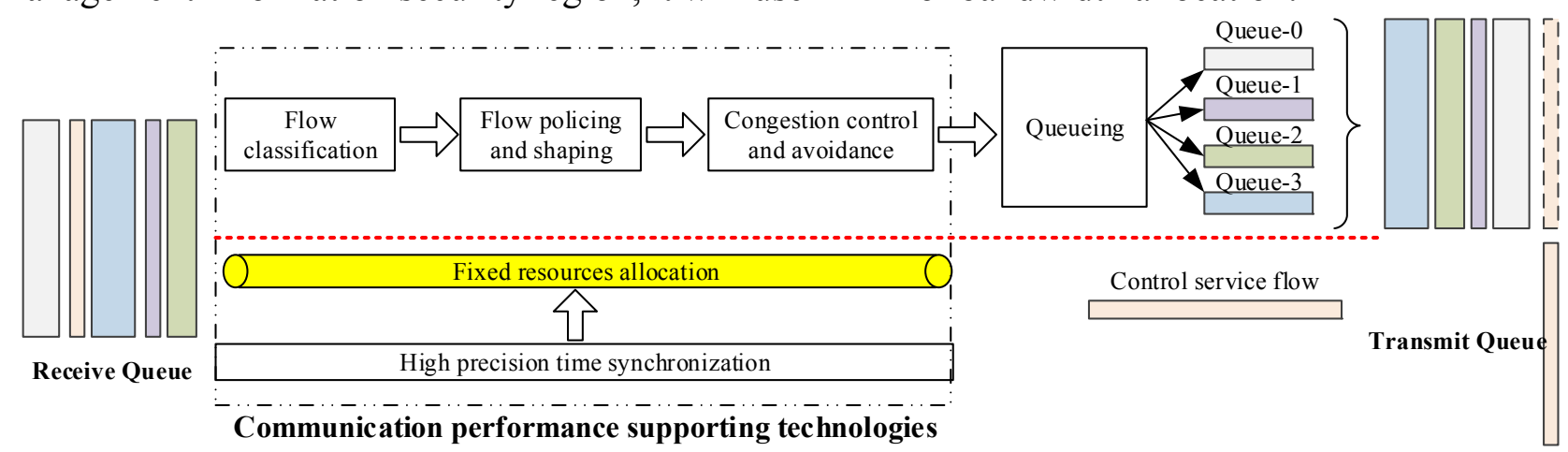

Fig.4. The dataflow of EPON based QoS scheme

From the above scheme, we can guarantee communication performance of the important services of production control security region, and improve network resource utilization for bandwidth sharing of the service of management information security region.

\section{Comparison of the Current Network and the Unified Multi-service Bearing Network}

Make a comparison of the current network and the unified multi-service bearing network, we can get many conclusions. Firstly, the construction cost is reduced nearly a half. The reason is that there are two physical networks in current, but the new scheme can realize all service bearing in one physical access network. Secondly, utilization of the network resource is greatly improved. The reason is that the resource utilization is low in the current network because of the bandwidth of single service is far less than the network can provide., but the new network can improve it by multi-service bearing. Last but not the least, network reliability will enhance and new services deployment will more flexible. The reason is that the current network has complex and independent network structure, but the new scheme is all in one and unified.

\section{Simulation Verification}

In the simulation, we will mainly focus on communication performance influence between different service flows. It mainly contains two aspects: isolation and convergence.

For the service flow isolation test, the configuration diagram is shown in Fig5(a) Simulation configuration: service from different security region, which is including distribution automation and electricity consumption information collection. The simulation result is electricity consumption information collection service flow in case of $40 \%, 60 \%, 80 \%, 100 \%$, the communication service of distribution automation is not affected by anyway.

For the service flow convergence test, the configuration diagram is shown in Fig5(b) Simulation 
configuration: service from different security region, which is including distribution automation, distributed power access $(\geqslant 200 \mathrm{~kW})$ and electricity consumption information collection corresponding to ONU1, ONU2 and ONU3. The simulation result is the packet lost rate of ONU3 is increasing with the service flow in the normal flow range, but ONU1 and ONU2 are not.

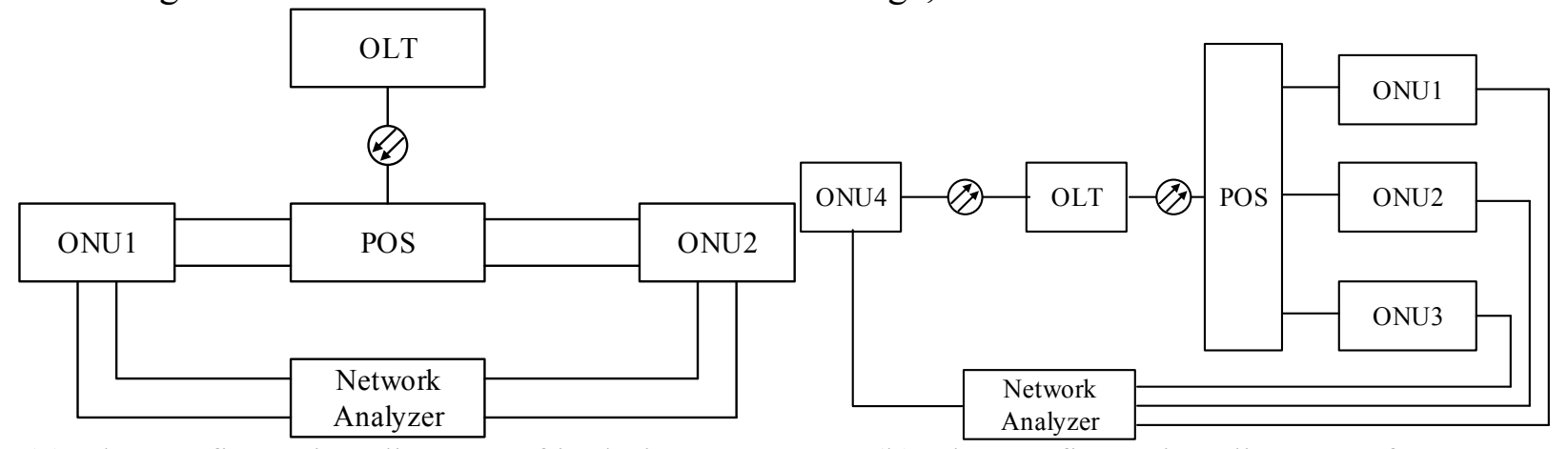

(a) The configuration diagram of isolation

(b) The configuration diagram of convergence

Fig.5. The simulation verification configuration

From simulation verification, we can find communication performance of production control security region can be guaranteed by fixed time slot. So the proposed scheme can realize multi-service isolation and QoS of different services.

\section{Conclusion}

In this paper, based on the analysis of the current electric power communication access network and key supporting technology of multi-service bearing network, we proposed a novel multi-service unified bearing network architecture. And implementation scheme based on EPON is also put forward. The simulation result revels that the proposed scheme is effective. It can solve problem of the current network and it is an evolution direction of the future.

\section{Acknowledgement}

In this paper, the research was sponsored by the Science and Technology Project of Tianjin Electric Power Company (Project Name: Research on key technologies of multi-service access communication for smart grid).

\section{References}

[1] J.H. Guo. Study on the structure of electric power communication network of strong and smart grid in China [A]. IEEE Computer Society, POWERCON2010[C]. 2010.

[2] B. Feng, Y.L. Lin, Y. Li, and so on. A novel method of requirement analysis for power communication access network [A]. Advanced Materials Research [C]. 2014(986-987): 2091-2094.

[3] Z.W. Sun. A survey on EPON-based communication networks for smart grid [A]. Adv. Ma-ter. Res[C]. 2013(765-767): 2633-2636.

[4] W.G. Liu, Y.L. Lin, B. Feng, and so on. Research on the convergence trend of power communication network [A]. Advanced Materials Research [C]. May 2014(986-987): 2108-2112.

[5] J. Qi, N. Mohamed, X.B. Leng, and so on. Multi-service access platform based on EPON [A], SPIE[C]. 2003(5282): 758-762.

[6] N. Yaling. A multicast QoS Control scheme and system in EPON system[C], Inst. of Elec. and Elec. Eng. Computer Society, 2007.

[7] W. Chen, Z.J. Zhang, Y.H. Pan. Method to realize the QoS of a heterogeneous network based on EPON[C], Optical communication technology. 2015(9): 12-15. 